\title{
半选择性氧化形成三对二硫键合成利那洛肽
}

\author{
葛巍巍 $^{a, b}$ 陈 静 ${ }^{a}$ 张 也 ${ }^{a}$ 宗 良 ${ }^{a}$ 张 鸣 ${ }^{a}$ 董俊军*, \\ $\left({ }^{a}\right.$ 中国人民解放军防化学院生化防护系 北京 102205) \\ ( ${ }^{b}$ 公安消防部队高等专科学校训练部 昆明 650208)
}

\begin{abstract}
摘要 利用 Fmoc 固相合成策略, Wang 树脂为载体, 使用三苯甲基(Trt)和乙酰胺甲基(Acm)保护基的半胱氨酸合成了 3 条 $[4 \mathrm{Trt}+2 \mathrm{Acm}]$ 和 3 条 $[2 \mathrm{Trt}+4 \mathrm{Acm}]$ 利那洛肽的线性前体化合物. 在此基础上, 采用半选择性氧化策略合成含有三对 二硫键的利那洛肽.首先使用含三氟乙酸(TFA)的裂解剂脱除线性前体肽中半胱氨酸的 Trt 保护基, 并使用氯化血红素 催化氧化半胱氨酸自由颈基形成二硫键. 下一步使用 $\mathrm{PhS}(\mathrm{O}) \mathrm{Ph} / \mathrm{CH}_{3} \mathrm{SiCl}_{3}$ 试剂体系脱除剩余保护半胱氨酸的 $\mathrm{Acm}$ 保护 基，并同时形成二硫键. 使用这种策略，在 6 条线性前体肽中，有 3 条可以得到利那洛肽，转化率分别为 $71.9 \% 、 31.5 \%$ 、 $81.4 \%$. 通过分析 6 条线性前体肽中二硫键形成的先后顺序对目标产物生成的影响, 发现二硫键 Cys5-Cys13 的形成对 利那洛肽的氧化折叠非常关键, 在选择性氧化合成利那洛肽时应当优先形成这对二硫键.
\end{abstract}

关键词＼cjkstart利那洛肽；二硫键；氧化；半选择性

\section{Semiregioselective Formation of Linaclotide with Orthogonal Cysteine Protection Strategy}

\author{
Ge, Weiwei ${ }^{a, b}$ \\ Chen, Jing ${ }^{a}$ \\ Zhang, $\mathrm{Ye}^{a}$ \\ Zong, Liang ${ }^{a}$ \\ Zhang, Ming ${ }^{a}$ \\ Dong, Junjun*,a \\ ( ${ }^{a}$ Department of Bio-Chem Defence, Institute of Nuclear, Biological and Chemical Defence, Beijing 102205) \\ ( ${ }^{b}$ Department of Training, Public Security Fire Forces College, Kunming 650208)
}

\begin{abstract}
Six linear precursors of linaclotide containing different protected cysteine residues were synthesized by Fmoc solid-phase methods. Wang resin was used in the peptide syntheses. The protective groups of cysteine thiol were trityl (Trt) and acetamidomethyl $(\mathrm{Acm})$ in the different positions. The six linear precursors of linaclotide include three [4 Trt $+2 \mathrm{Acm}]$ and three $[2 \mathrm{Trt}+4 \mathrm{Acm}]$ ones. The linaclotide with three disulfide bonds was prepared from these linear precursors by semiregioselective strategy. Firstly, linear peptides were cleaved from Wang resins by TFA-TIS- $\mathrm{H}_{2} \mathrm{O}$. At the same time, the Trt groups were removed to give free thiol groups, whereas Acm groups were still remained in the peptides. Secondly, the free thiol groups were oxidized by $20 \%$ hemin/DIEA system to from disulfide bond(s). Finally, cysteines containing Acm groups were deprotected by $\mathrm{CH}_{3} \mathrm{SiCl}_{3} / \mathrm{PhS}(\mathrm{O}) \mathrm{Ph} / \mathrm{TFA}$ coaktail and disulfide bond(s) were formed simultaneously. The precursors of peptides $[4 \operatorname{Trt}(2,5,10,13)+2 \operatorname{Acm}(1,6)],[2 \operatorname{Trt}(1,6)+4 \operatorname{Acm}(2,5,10,13)]$ and $[2 \operatorname{Trt}(5,13)+2 \operatorname{Acm}(1,2,6,10)]$ give linaclotide at the conversion ratios of $71.9 \%, 31.5 \%$, and $81.4 \%$ respectively. Other three peptides failed in the conversion or were found to be less suitable to prepare linaclotide. Our results indicated that the order of disulfide bond formation is very important to prepare linaclotide by using semiregioselective or regioselective strategies. The Cys5-Cys13 disulfide bond is the most privileged and should be formed firstly among the three disulfide bonds in linaclotide.
\end{abstract}

Keywords linaclotide; disulfide; oxidation; semiregioselective

利那洛肽(linaclotide)是首个鸟苷酸环化酶(GC)激 动剂类药物 ${ }^{[1]}$, 口服进入人体后通过与肠道中的鸟苷酸 环化酶 C 型受体 (GC-C)结合, 使细胞内和细胞外环鸟苷
酸(cGMP)浓度升高，刺激肠液分泌，加快胃肠道移行， 从而增加排便频率 ${ }^{[2,3]} .2012$ 年, 利那洛肽被美国食品和 药物管理局(FDA)批准可以用于治疗成人慢性特发性便

\footnotetext{
* Corresponding author. E-mail: dongjj536@sina.com

Received April 12, 2017; revised May 15, 2017; published online May 24, 2017.

Project supported by the State Key Laboratory of Toxicology and Medical Countermeasures of China (No. PMC201507) and the State Key Laboratory of Nuclear, Biological and Chemical Protection for Civilian (No. SKLNBC2013-01K).

毒药物与毒理学国家重点实验室开放基金(No. PMC201507)和国民核生化灾害防护国家重点实验室开放基金(No. SKLNBC2013-01K)资助项目.
} 
秘和便秘型肠易激综合症(IBS-C), 由于其独一无二的 作用机制, 很有希望成为一种不但可以治疗便秘, 还可 以治疗腹胀、腹痛和其它临床症状的治疗药物. 利那洛 肽为含有三对二硫键的十四肽, 氨基酸组成为: CCEYCCNPACTGCY, 其肽链中的二硫键连接方式为 Cys1-Cys6, Cys2-Cys10, Cys5-Cys13. 目前, 对利那洛 肽的合成主要以自由氧化法形成三对二硫键为主, 选择 性氧化合成利那洛肽的研究很少.

乙酰胺甲基 (Acm)和三苯甲基( $\mathrm{Trt}$ ) 是最为常见的两 种可以互相正交保护的半胱氨酸保护基，常用于选择性 氧化合成含多对二硫键的多肽 ${ }^{[4]}$. 半胱氨酸上的 $\operatorname{Trt}$ 保 护基在用三氟乙酸(TFA)裂解树脂时可以与其它氨基酸 的保护基一并脱除, 在此条件下 Acm 保护基则不受影 响, 在下一步反应中可以用 $\mathrm{I}_{2}$ 或重金属盐 $\left(\mathrm{Hg}^{2+} 、 \mathrm{Tl}^{3+}\right.$ 、 $\mathrm{Ag}^{+}$) 的方法, 脱去 $\mathrm{Acm}$ 的同时氧化形成二硫键 ${ }^{[7 \sim 10]}$. Miriam 等 ${ }^{[11]}$ 在对利那洛肽的合成策略研究中合成了 3 条 [4 Trt $+2 \mathrm{Acm}]$ 和 2 条 $[2 \mathrm{Trt}+4 \mathrm{Acm}]$ 利那洛肽的线性 前体化合物, 先采用 TFA 脱除 Trt, DMSO 氧化的方法形 成了部分二硫键, 但下一步 $\mathrm{I}_{2}$ 脱除 $\mathrm{Acm}$ 的反应却没有成 功, 即使后来作者将氧化条件变为在低温下缓慢滴加 $\mathrm{I}_{2}$ (以减慢氧化环合的速度), 仍然没有得到目标多肽. 文章中, Miriam 等还合成了 2 条 $[2 \mathrm{Mmt}+2 \mathrm{Acm}+2 \mathrm{Trt}]$ 和 2 条 $[2 \mathrm{Acm}+2 \mathrm{Trt}+2 \mathrm{pMeOBzl}]$ 利那洛肽的线性前体 化合物, 拟采用选择性氧化的方法形成三对二硫键, 但 均没有得到目标多肽, 因此 Miriam 认为 Acm 保护的半 胱氨酸不适用于利那洛肽的合成.

本文利用 Fmoc 固相合成策略, Wang 树脂为载体合 成了 3 条 $[4 \mathrm{Trt}+2 \mathrm{Acm}]$ 和 3 条 $[2 \mathrm{Trt}+4 \mathrm{Acm}]$ 利那洛肽 的线性前体化合物, 在裂解树脂的同时脱去半胱氨酸上 的 Trt 保护基, 使用氯化血红素催化氧化的方法形成部 分二硫键 ${ }^{[2]}$. 下一步反应, 使用了二苯亚砜 $[\mathrm{PhS}(\mathrm{O}) \mathrm{Ph}] /$ 三甲基氯硅烷 $\left(\mathrm{CH}_{3} \mathrm{SiCl}_{3}\right)$ 体系 ${ }^{[13]}$, 在 TFA 中脱除 $\mathrm{Acm}$ 保 护基并形成剩余的二硫键. 合成的 6 条线性前体肽中, 有 3 条可以较高转化率得到利那洛肽, 成功的将 Acm 保 护的半胱氨酸应用于利那洛肽的合成中. 通过分析 6 条 线性前体肽中二硫键形成的先后顺序对目标多肽生成 的影响, 发现 Cys5-Cys13 二硫键的形成对利那洛肽的 氧化折叠非常关键, 在选择性氧化合成利那洛肽时应当 优先形成这对二硫键.

\section{1 结果与讨论}

\section{1 合成 3 条利那洛肽前体化合物[4 $\mathrm{Trt}+2 \mathrm{Acm}]$}

利用 Fmoc 固相合成策略, Wang 树脂为载体, 合成 了 3 条 $[4 \mathrm{Trt}+2 \mathrm{Acm}]$ 保护的利那洛肽线性前体化合物 (图 1).

\subsection{1一步氧化形成两对二硫键}

将多肽树脂放入反应瓶中裂解, 乙醚沉淀得到粗 肽, 粗肽分离纯化后进行质谱检测, $\mathbf{a}\left([\mathrm{M}+\mathrm{H}]^{+}=\right.$ 1674.8, $\left.[\mathrm{M}+\mathrm{Na}]^{+}=1696.8,[\mathrm{M}+\mathrm{K}]^{+}=1712.8\right), \mathbf{b}([\mathrm{M}+$ $\left.\mathrm{H}]^{+}=1674.9,[\mathrm{M}+\mathrm{Na}]^{+}=1696.9,[\mathrm{M}+\mathrm{K}]^{+}=1712.9\right), \mathbf{c}$ $\left([\mathrm{M}+\mathrm{H}]^{+}=1674.7,[\mathrm{M}+\mathrm{Na}]^{+}=1696.7,[\mathrm{M}+\mathrm{K}]^{+}=\right.$ 1712.7), 利那洛肽 $[4 \mathrm{SH}+2 \mathrm{Acm}]$ 的线性前体肽理论分 子量为 1673.5, 证明得到的多肽结构为 [4 SH+2 Acm]. 使用氯化血红素催化氧化的方法分别氧化 $\mathbf{a} 、 \mathbf{b} 、 \mathbf{c}$, 其 中 $\mathbf{a} 、 \mathbf{b}$ 氧化可以得到一个主产物 $\mathbf{a}-1 、 b-1$, 由于 $\mathbf{c}$ 的氧 化产物过于复杂(图 2), 没有对其分离及进行下一步反 应. 分别对 a-1、b-1 进行分离纯化, 质谱检测 a-1 $\left([\mathrm{M}+\mathrm{Na}]^{+}=1692.4,[\mathrm{M}+\mathrm{K}]^{+}=1708.4\right), \mathbf{b}-1 \quad([\mathrm{M}+$ $\left.\mathrm{H}]^{+}=1670.5,[\mathrm{M}+\mathrm{Na}]^{+}=1692.5,[\mathrm{M}+\mathrm{K}]^{+}=1708.4\right)$, 证明其结构为 $[2 \mathrm{~S}-\mathrm{S}+2 \mathrm{Acm}]$.

\subsection{2 氧化形成第三对二硫键}

在下一步脱除 Acm 保护基形成二硫键的反应中, 我们首先尝试了 $\mathrm{I}_{2}$ 氧化脱除的方法，但与文献报道一 样，都没有得到目标产物。如前体肽 a-1 [2 S-S(2-10, $5-13)+2 \operatorname{Acm}(1,6)]$, 只含有一对 $\mathrm{Acm}$ 保护基, 理论上脱 Acm 后 HPLC 分析应该为一个单峰, 但滴加 $\mathrm{I}_{2}$ 氧化后却 生成了三个产物, 且都不是目标多肽. 随后我们采用了 $\mathrm{PhS}(\mathrm{O}) \mathrm{Ph} / \mathrm{CH}_{3} \mathrm{SiCl}_{3}$ 氧化的方法, 将上面得到的 a-1 溶于 TFA 中, 加入过量的 $\mathrm{CH}_{3} \mathrm{SiCl}_{3}$ 和 $\mathrm{PhS}(\mathrm{O}) \mathrm{Ph}$ 摚拌反应 20 $\min$, 加入乙醚和 $4 \mathrm{~mol} / \mathrm{L}$ 的醋酸震荡, 取水相与 $\mathbf{a}-\mathbf{1}$ 、 利那洛肽标准品共同进样 HPLC 分析. 从色谱图上看, $\mathbf{a}-1$ 反应完全，几乎定量得到单一的产物 $\mathbf{a}-\mathbf{2}, \mathbf{a}-\mathbf{2}$ 的色谱 行为与标准品一致(图 3). 将 a-2 分离纯化后进行质谱检 测, a-2 $\left([\mathrm{M}+\mathrm{H}]^{+}=1526.6,[\mathrm{M}+\mathrm{Na}]^{+}=1548.6,[\mathrm{M}+\right.$ $\mathrm{K}]^{+}=1564.6$ ), 利那洛肽的理论分子量为 1525.4 , 与理 论值相符，可以证明 $\mathbf{a}-\mathbf{2}$ 为利那洛肽，同时也证明了 $\mathbf{a}-\mathbf{1}$ 中的两对二硫键连接方式为 Cys2-Cys10, Cys5-Cys13.

在其它条件不变情况下, 将 $\mathbf{a}-\mathbf{1}$ 与 $\mathrm{PhS}(\mathrm{O}) \mathrm{Ph}$ 、 $\mathrm{CH}_{3} \mathrm{SiCl}_{3}$ 反应的时间延长至 $30 \mathrm{~min}, \mathrm{HPLC}$ 分析结果相 同，说明反应没有二硫键重排现象发生 ${ }^{[14]}, \mathrm{PhS}(\mathrm{O}) \mathrm{Ph} /$ $\mathrm{CH}_{3} \mathrm{SiCl}_{3}$ 氧化的方法可以用于脱除利那洛肽的线性前 体肽中半胱氨酸的 $\mathrm{Acm}$ 保护基, 并氧化形成二硫键.

然而，在使用相同的方法脱除 b-1 上半胱氨酸的 Acm 保护基时, HPLC 分析发现 b-1 与 $\mathrm{PhS}(\mathrm{O}) \mathrm{Ph} /$ $\mathrm{CH}_{3} \mathrm{SiCl}_{3}$ 几乎不反应，我们在开始尝试用 $\mathrm{I}_{2}$ 脱除 b-1 中 的 Acm 保护基时也发现存在同样的现象. 说明 b-1 中的 两对二硫键形成的特定空间结构, 大大降低了 Cys2 和 Cys10 之间相互接触发生反应的几率，使其不能顺利脱 除 $\mathrm{Acm}$ 保护基而形成二硫键. 
(a) H-Cys(Acm)-Cys(Trt)-Glu(t-Bu)-Tyr(t-Bu)-Cys(Trt)-Cys(Acm)-Asn(Trt)-Pro-Ala-Cys(Trt)-Thr-Gly-Cys(Trt)-Tyr(t-Bu)-Resin

(b) H-Cys(Trt)-Cys(Acm)-Glu(t-Bu)-Tyr(t-Bu)-Cys(Trt)-Cys(Trt)-Asn(Trt)-Pro-Ala-Cys(Acm)-Thr-Gly-Cys(Trt)-Tyr(t-Bu)-Resin

(c) H-Cys(Trt)-Cys(Trt)-Glu(t-Bu)-Tyr(t-Bu)-Cys(Acm)-Cys(Trt)-Asn(Trt)-Pro-Ala-Cys(Trt)-Thr-Gly-Cys(Acm)-Tyr(t-Bu)-Resin

图 1 三条 $[4 \mathrm{Trt}+2 \mathrm{Acm}]$ 保护的利那洛肽线性前体化合物

Figure 1 Three linear precursors of linaclotide containing [4 Trt $+2 \mathrm{Acm}]$ groups
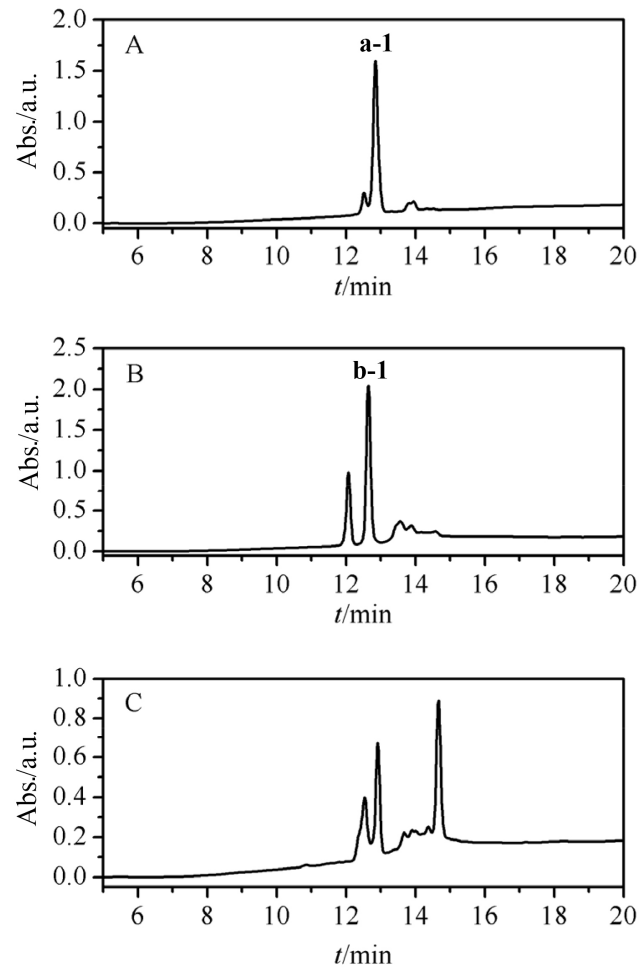

图 2 三条 $[4 \mathrm{Trt}+2 \mathrm{Acm}]$ 利那洛肽的线性前体肽脱除 $\mathrm{Trt}$ 保护 后一步氧化形成两对二硫键 HPLC 分析

Figure 2 After removing Trt from [4 Trt +2 Acm] linear precursors of three linaclotides were oxidized to form two disulfide bonds

(A) a $[4 \mathrm{SH}(2,5,10,13)+2 \mathrm{Acm}(1,6)]$, (B) b $[4 \mathrm{SH}(1,5,6,13)+2$ $\operatorname{Acm}(2,10)],(C)$ c $[4 \mathrm{SH}(1,2,5,10)+2 \mathrm{Acm}(5,13)]$

\section{2 合成 3 条利那洛肽前体化合物[2 Trt+4 Acm]}

使用第 1.1 节中相同的方法合成了 3 条 $[2 \mathrm{Trt}+4$ Acm]保护的利那洛肽线性前体化合物(图 4).

\section{2 .1 氧化形成第一对二硫键}

使用第 1.1.1 节中的相同方法裂解树脂, 得到含自 由䘪基的利那洛肽线性前体肽 $\mathbf{d} 、 \mathbf{e} 、 \mathbf{f}$ ，质谱检测 $\mathbf{d}$ $\left([\mathrm{M}+\mathrm{H}]^{+}=1816.9,[\mathrm{M}+\mathrm{Na}]^{+}=1839.0,[\mathrm{M}+\mathrm{K}]^{+}=\right.$ 1855.0), e $\left([\mathrm{M}+\mathrm{Na}]^{+}=1838.6,[\mathrm{M}+\mathrm{K}]^{+}=1854.6\right), \mathbf{f}$ $\left([\mathrm{M}+\mathrm{H}]^{+}=1816.7,[\mathrm{M}+\mathrm{Na}]^{+}=1838.7,[\mathrm{M}+\mathrm{K}]^{+}=\right.$

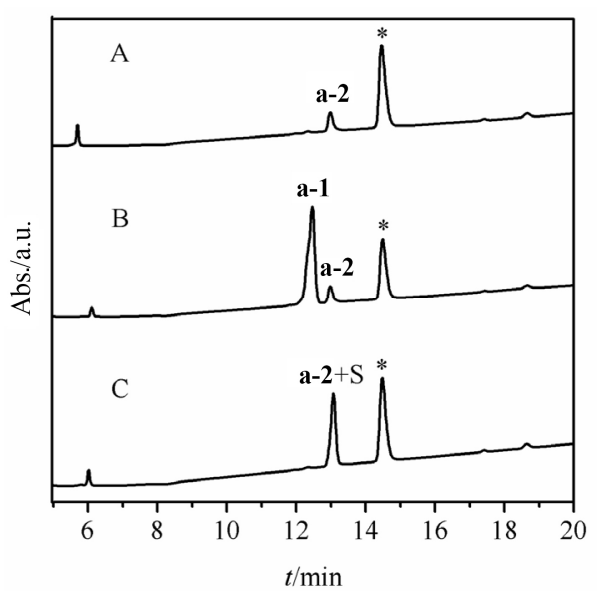

图 3 a-1 [2 S-S(2-10,5-13)+2 Acm(1,6)]采用 $\mathrm{PhS}(\mathrm{O}) \mathrm{Ph} /$ $\mathrm{CH}_{3} \mathrm{SiCl}_{3}$ 体系氧化后 HPLC 分析(*为杂质峰)

Figure 3 HPLC analysis of a-1 [2 S-S(2-10,5-13) + 2 $\operatorname{Acm}(1,6)]$ after treatment of $(\mathrm{PhS}(\mathrm{O}) \mathrm{Ph}) /\left(\mathrm{CH}_{3} \mathrm{SiCl}_{3}\right)$

(A) a-1 after treatment of $(\mathrm{PhS}(\mathrm{O}) \mathrm{Ph}) /\left(\mathrm{CH}_{3} \mathrm{SiCl}_{3}\right),(\mathrm{B})$ mixture of $\mathrm{A}$ and a-1, (C) mixture of A and standard linaclotide (S). The peak * (retention time at $14.5 \mathrm{~min}$ ) LCMS observed $[\mathrm{M}+\mathrm{H}]^{+}=247$

1854.7), 利那洛肽 $[2 \mathrm{SH}+4 \mathrm{Acm}]$ 的线性前体肽理论分 子量为 1815.5 , 证明制得的多肽结构为 [2 $\mathrm{SH}+4 \mathrm{Acm}]$. 在下一步使用氯化血红素催化氧化形成二硫键时, 由于 d、e、f 只含有一对自由的颈基, HPLC 分析几乎都得到 了唯一的产物: d-1、e-1、f-1, 分别将其进行分离纯化进 行质谱分析, d-1 $\left([\mathrm{M}+\mathrm{H}]^{+}=1813.4,[\mathrm{M}+\mathrm{Na}]^{+}=1836.6\right.$, $\left.[\mathrm{M}+\mathrm{K}]^{+}=1852.5\right), \mathbf{e}-1\left([\mathrm{M}+\mathrm{H}]^{+}=1814.7,[\mathrm{M}+\mathrm{Na}]^{+}=\right.$ 1836.6, $\left.[\mathrm{M}+\mathrm{K}]^{+}=1852.6\right), \mathbf{f}-1 \quad\left([\mathrm{M}+\mathrm{Na}]^{+}=1836.7\right.$, $\left.[\mathrm{M}+\mathrm{K}]^{+}=1852.7\right)$, 证明其结构为 $[1 \mathrm{~S}-\mathrm{S}+4 \mathrm{Acm}]$.

\section{2 .2 一步氧化形成剩余两对二硫键}

使用 $(\mathrm{PhS}(\mathrm{O}) \mathrm{Ph}) /\left(\mathrm{CH}_{3} \mathrm{SiCl}_{3}\right)$ 方法一步氧化 d-1、e-1、 $\mathbf{f - 1}$, 加乙醚、醋酸震荡后取水相进行 HPLC 分析, 发现 d-1 氧化得到两个主产物 d-2、d-3, 色谱峰面积比为 57: 43(图 6, A); e-1 氧化产物复杂, 没有明显的主产物; $\mathbf{f - 1}$ 氧化得到一个主产物 f-2(图 7,A).

将 d-1 氧化反应的水相与 d-1、利那洛肽标准品共 进样 HPLC 分析, 发现 d-3 的色谱行为与标准品一致(图

(d) H-Cys(Trt)-Cys(Acm)-Glu(t-Bu)-Tyr(t-Bu)-Cys(Acm)-Cys(Trt)-Asn(Trt)-Pro-Ala-Cys(Acm)-Thr-Gly-Cys(Acm)-Tyr(t-Bu)-Resin (e) H-Cys(Acm)-Cys(Trt)-Glu(t-Bu)-Tyr(t-Bu)-Cys(Acm)-Cys(Acm)-Asn(Trt)-Pro-Ala-Cys(Trt)-Thr-Gly-Cys(Acm)-Tyr(t-Bu)-Resin (f) H-Cys(Acm)-Cys(Acm)-Glu(t-Bu)-Tyr(t-Bu)-Cys(Trt)-Cys(Acm)-Asn(Trt)-Pro-Ala-Cys(Acm)-Thr-Gly-Cys(Trt)-Tyr(t-Bu)-Resin

图 4 三条 $[2 \mathrm{Trt}+4 \mathrm{Acm}]$ 保护的利那洛肽线性前体化合物

Figure 4 Three linear precursors of linaclotide containing [2 Trt $+4 \mathrm{Acm}]$ groups 

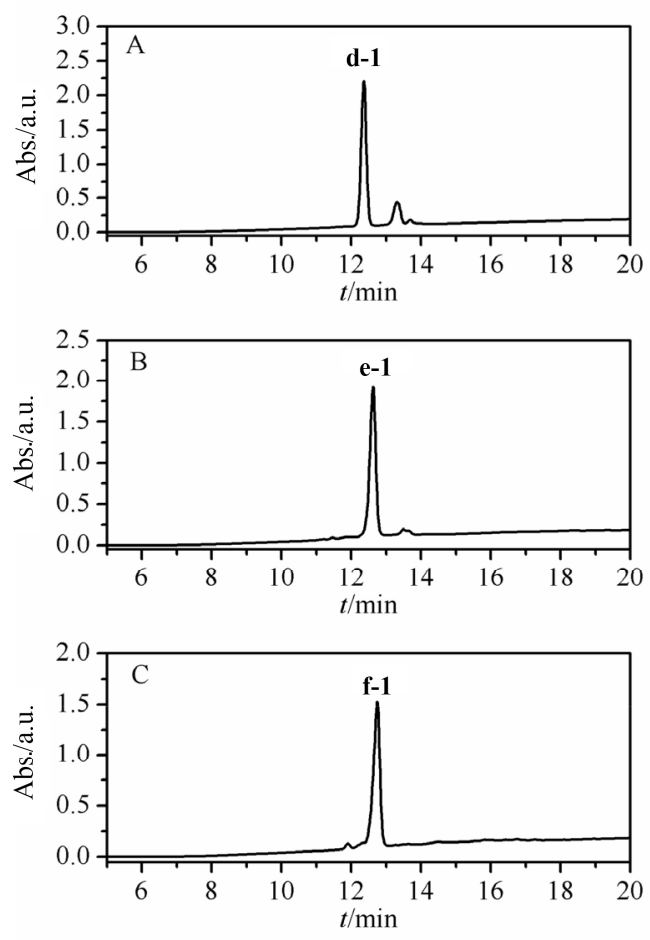

图 5 三条 $[2 \mathrm{Trt}+4 \mathrm{Acm}]$ 利那洛肽的线性前体肽脱除 Trt 保护 后氧化形成第一对二硫键 HPLC 分析

Figure 5 After removing Trt from [2 $\mathrm{Trt}+4 \mathrm{Acm}]$ linear precursors of linaclotide, were oxidized to form one disulfide bond (A) d $[2 \mathrm{SH}(1,6)+4 \mathrm{Acm}(2,5,10,13)]$, (B) e $[2 \mathrm{SH}(2,10)+4$ $\operatorname{Acm}(1,5,6,13)],(\mathrm{C}) \mathbf{f}[2 \mathrm{SH}(5,13)+4 \operatorname{Acm}(1,2,6,10)]$

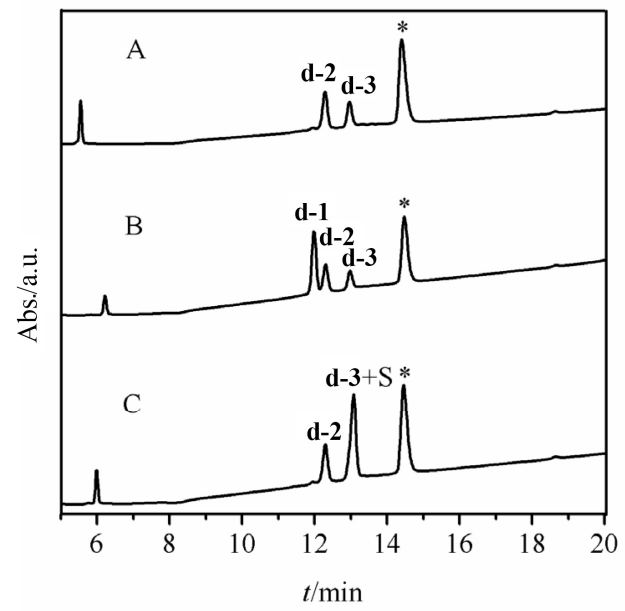

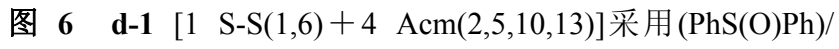
$\left(\mathrm{CH}_{3} \mathrm{SiCl}_{3}\right)$ 体系氧化后 HPLC 分析(*为杂质峰)

Figure 6 HPLC analysis of d-1 $[2 \mathrm{~S}-\mathrm{S}(1,6)+4 \mathrm{Acm}(2,5,10,13)]$ after treatment of $(\mathrm{PhS}(\mathrm{O}) \mathrm{Ph}) /\left(\mathrm{CH}_{3} \mathrm{SiCl}_{3}\right)$

(A) d-1 after treatment of $(\mathrm{PhS}(\mathrm{O}) \mathrm{Ph}) /\left(\mathrm{CH}_{3} \mathrm{SiCl}_{3}\right),(\mathrm{B})$ mixture of $\mathrm{A}$ and d-1, (C) mixture of A and standard linaclotide(S). The peak* (retention time at $14.5 \mathrm{~min}$ ) LCMS observed $[\mathrm{M}+\mathrm{H}]^{+}=247$

6, B、C), 将 d-2, d-3 分离纯化后进行质谱检测, d-2 $\left([\mathrm{M}+\mathrm{H}]^{+}=1526.5,[\mathrm{M}+\mathrm{Na}]^{+}=1548.6,[\mathrm{M}+\mathrm{K}]^{+}=\right.$ $1564.5)$, d-3 $\left([\mathrm{M}+\mathrm{H}]^{+}=1526.5,[\mathrm{M}+\mathrm{Na}]^{+}=1548.5\right.$,

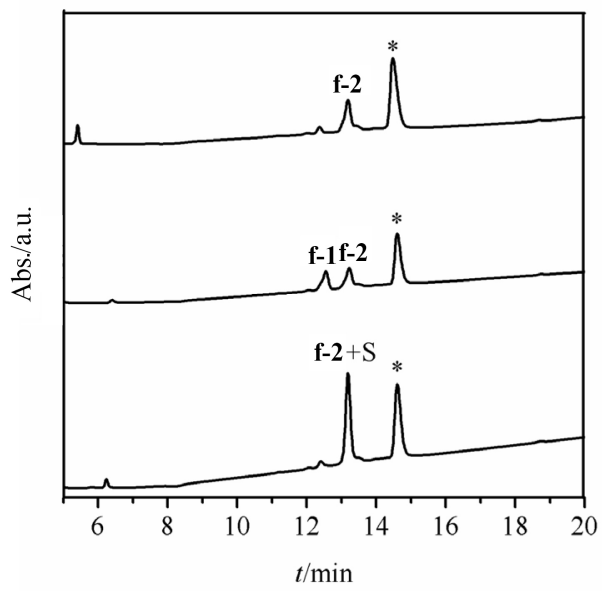

图 7 f-1 $[1 \quad \mathrm{~S}-\mathrm{S}(5,13)+4 \mathrm{Acm}(1,2,6,10)]$ 采用 $\mathrm{PhS}(\mathrm{O}) \mathrm{Ph} /$ $\mathrm{CH}_{3} \mathrm{SiCl}_{3}$ 体系一步氧化后 HPLC 分析(*为杂质峰)

Figure 7 HPLC analysis of (A) f-1 [1 S-S $(5,13)+4$ Acm $(1,2,6$, 10)] after treatment of $(\mathrm{PhS}(\mathrm{O}) \mathrm{Ph}) /\left(\mathrm{CH}_{3} \mathrm{SiCl}_{3}\right)$

f-1 after treatment of $(\mathrm{PhS}(\mathrm{O}) \mathrm{Ph}) /\left(\mathrm{CH}_{3} \mathrm{SiCl}_{3}\right)$, (B) mixture of $\mathrm{A}$ and $\mathbf{f}-\mathbf{1}$, (C) mixture of A and standard linaclotide (S). The peak* (retention time at 14.5 $\mathrm{min}$ ) LCMS observed $[\mathrm{M}+\mathrm{H}]^{+}=247$

$\left.[\mathrm{M}+\mathrm{K}]^{+}=1564.4\right)$ 都与利那洛肽的理论分子量相符, 证 明 d-2 为利那洛肽的二硫键错配异构体, d-3 为利那洛 肽.

将 $\mathbf{f - 1}$ 氧化反应的水相与 $\mathbf{f - 1}$ 、利那洛肽标准品共同 进样 HPLC 分析, 发现 $\mathbf{f - 2}$ 的色谱行为与标准品一致(图 $7, \mathrm{~B} 、 \mathrm{C})$, 将 $\mathbf{f - 2}$ 分离纯化后质谱检测, $\mathbf{f}-2\left([\mathrm{M}+\mathrm{H}]^{+}=\right.$ $\left.1526.5,[\mathrm{M}+\mathrm{Na}]^{+}=1548.5,[\mathrm{M}+\mathrm{K}]^{+}=1564.5\right)$ 与利那洛 肽的理论分子量相符, 证明 $\mathbf{f - 2}$ 为利那洛肽.

\section{3 讨论}

从本文的实验结果来看, 二硫键形成的顺序对利那 洛肽的氧化折叠结果影响很大，可以直接决定能否得到 目标多肽. 合成的 6 条利那洛肽线性前体肽中, 产率较 高两条 a $[4 \operatorname{Trt}(2,5,10,13)+2 \operatorname{Acm}(1,6)]$ 和 f $[2 \operatorname{Trt}(5,13)$ $+4 \operatorname{Acm}(1,2,6,10)]$ 都是先形成的 Cys5-Cys13; 而最后形 成 Cys5-Cys13 的线性前体肽 c $[4 \operatorname{Trt}(1,2,6,10)+2$ $\operatorname{Acm}(5,13)]$ 则在第一步氧化反应中就生成了比较复杂的 产物, 说明二硫键 Cys5-Cys13 的形成在利那洛肽氧化 折叠过程中非常关键.

Miriam 合成了 2 条利那洛肽的线性前体肽 $[2 \operatorname{Trt}(2$, $10)+2 \operatorname{Acm}(1,6)+2 p \operatorname{peOBzl}(5,13)]$ 和 $[2 \operatorname{Trt}(5,13)+2$ $\operatorname{Acm}(1,6)+2 \mathrm{pMeOBzl}(2,10)]$ 在脱除了半胱氨酸的 Trt 保 护基，氧化形成第一对二硫键时，第 1 条肽可以形成 Cys2-Cys10, 而第 2 条肽无法形成 Cys5-Cys13, 因此 Miriam 认为二硫键 Cys5-Cys13 对利那洛肽的氧化折叠 没有帮助. 但本文中合成的利那洛肽线性前体肽 d、e、 f 在氧化形成第一对二硫键时, 均能得到第一对二硫键 在不同位置的产物 d-1 (Cys1-Cys6)、e-1 (Cys2-Cys10)、 
f-1 (Cys5-Cys13). 产生上述差异的原因, 可能是由于 4甲氧基苄基(pMeOBzl)的空间位阻作用使得 Miriam 合 成的线性前体肽中的 Cys5-Cys13 难以形成, 因为该报 道中, 即使 $[2 \mathrm{SH}(2,10)+2 \mathrm{Acm}(1,6)+2 \mathrm{pMeOBzl}(5,13)]$ 形成了 Cys2-Cys10 这对二硫键, 也需要 DMSO 氧化 36 h.

此外, Miriam 认为利那洛肽中的二硫键 Cys1-Cys6 非常重要, 在合成时要优先形成. 文章中合成的利那洛 肽线性前体肽 $[2 \mathrm{StBu}(1,6)+2 \operatorname{Trt}(2,10)+2 \mathrm{pMeO}-$ Bzl $(5,13)]$ 按照先形成 Cys1-Cys6, 再形成 Cys2-Cys10、 Cys5-Cys13 的顺序选择性氧化得到了目标多肽(反应同 时还生成了几个二硫键错配的异构体). 本文中的线性 前体肽 d 也是先形成的 Cys1-Cys6, 然后脱除半胱氨酸 的 Acm 保护基并氧化形成剩余两对二硫键得到目标多 肽 $\mathbf{d - 3}$ (反应同时也生成一个转化率更高的二硫键错配 异构体 d-2), 这和 Miriam 的实验结果是相符的. 从本文 中线性前体肽 $\mathbf{a}, \mathbf{f}($ 优先形成 Cys5-Cys13)氧化生成目标 多肽的数据看, 经过两步氧化后, 线性前体肽 $\mathbf{a}$ 生成目 标产物的转化率达到 $71.9 \%$, 而线性前体肽 $\mathrm{f}$ 生成目标 产物的转化率达到 $81.4 \%$, 大大优于线性前体肽 $\mathrm{d}$ (优先 生成 Cys1-Cys6)的转化率 31.5\%. 因此, 在半选择性氧 化及选择性氧化合成利那洛肽时, 二硫键 Cys5-Cys13 应优先形成.

\section{2 结论}

本文运用半选择性氧化策略, Trt 和 Acm 保护的半 胱氨酸, 以 Wang 树脂为载体, 合成了 6 条不同的利那洛 肽线性前体化合物. 以此为基础，对利那洛肽的合成进 行研究. 首先使用含 TFA 的裂解剂脱除利那洛肽线性 前体肽中半胱氨酸的 Trt 保护基, 并使用氯化血红素催 化氧化的方法形成部分二硫键, 下一步使用 $\mathrm{PhS}(\mathrm{O}) \mathrm{Ph} /$ $\mathrm{CH}_{3} \mathrm{SiCl}_{3}$ 的方法脱除半胱氨酸的 $\mathrm{Acm}$ 保护基并同时氧 化形成剩余的二硫键，成功地将 Trt 和 Acm 两种正交保 护的半胱氨酸保护基用于利洛那肽合成中. 与文献中的 反应时间相比, 本文第一步氧化反应需要 $2 \mathrm{~h}$, 第二步 氧化反应需要 $20 \mathrm{~min}$, 大大缩短了反应时间.

合成的 6 条线性利那洛肽中, 有 3 条 (a、d、f)可以 得到利那洛肽, 转化率分别为 $71.9 \% 、 31.5 \% 、 81.4 \%$ (Scheme 1). 通过比较了二硫键形成顺序对氧化折叠效 果的影响, 发现二硫键 Cys5-Cys13 在利那洛肽氧化折 叠过程中非常关键. 本文中线性前体肽 $\mathrm{f}$ 先形成了二硫 键 Cys5-Cys13, 再一步氧化形成剩余的二硫键, 有效降 低了在一步氧化过程中的二硫键错配几率, 得到的利那 洛肽转化率高于我们在前期研究中使用自由氧化法合 成利那洛肽的转化率 $(75.7 \%)^{[15]}$, 可以为利那洛肽的合 成提供借鉴.

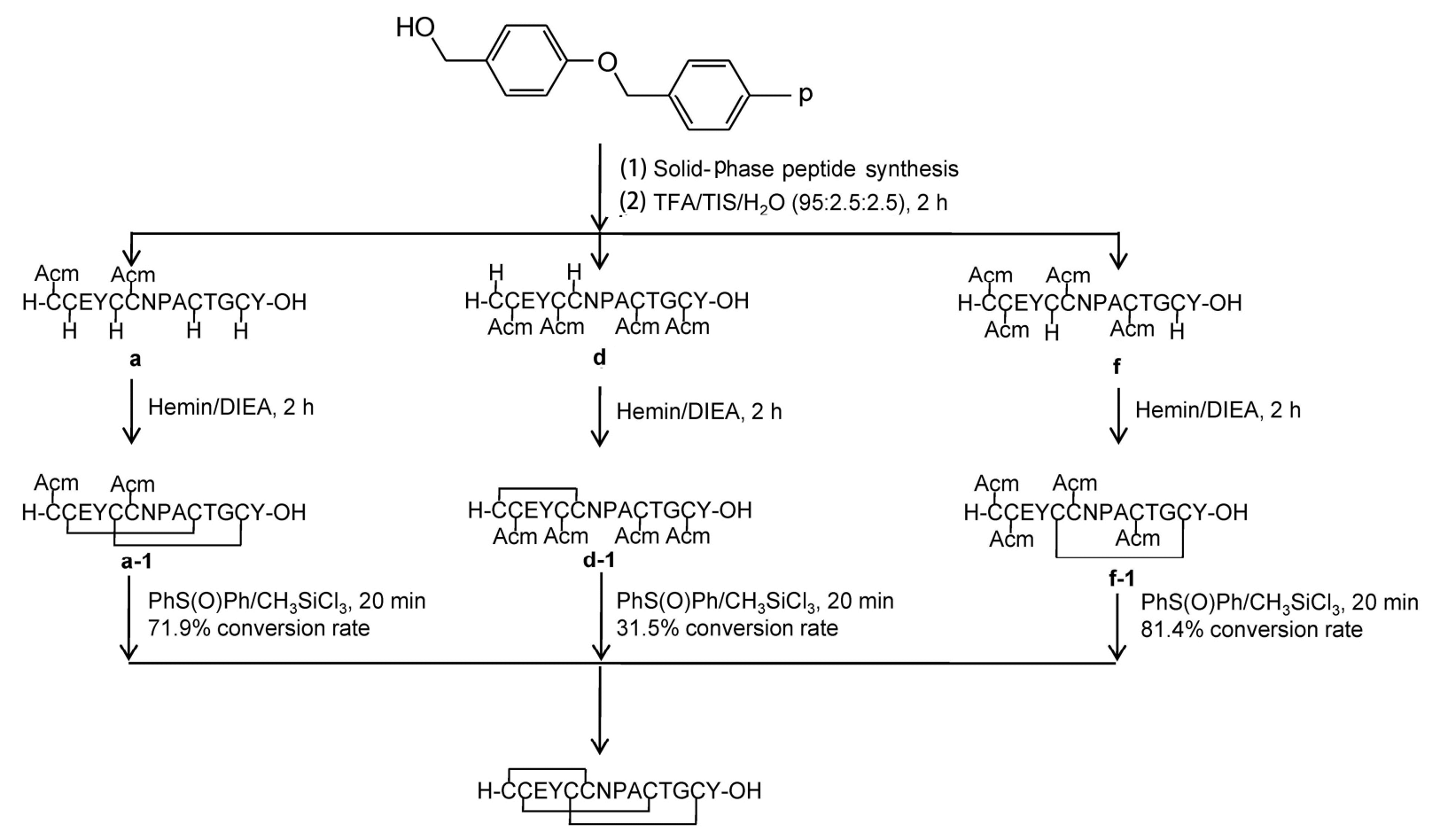

图式 1 半选择性策略氧化形成含三对二硫键的利那洛肽路线

Scheme 1 Semiregioselective strategy for linaclotide preparation 


\section{3 实验部分}

\section{1 仪器与试剂}

Waters 600 半制备液相, $\mathrm{C} 18$ 分析柱 $(3.9 \mathrm{~mm} \times 150$ $\mathrm{mm}, 5.0 \mu \mathrm{m}), \mathrm{XB}-\mathrm{C} 18$ 制备柱 $(21.2 \mathrm{~mm} \times 150 \mathrm{~mm}, 10.0$ $\mu \mathrm{m})($ 美国 Waters 公司); LP-3 型冷冻干燥机(法国 JOUAN 公司); BIFLEX III 型 MALDI-TOF 质谱仪(德国 Bruker 公司).

利那洛肽标准品(纯度 99\%), 成都凯捷生物医药科 技发展有限公司提供; 氯化血红素(纯度 $97 \%$ ), 购自天 津生命科学应用研究所; Wang 树脂, Fmoc-Cys(Trt), Fmoc-Cys(Acm), Fmoc-Glu(tBu), Fmoc-Tyr $(t \mathrm{Bu})$, FmocAsn(Trt), Fmoc-Pro, Fmoc-Ala, Fmoc-Thr(tBu), FmocGly, 1-羟基苯并三唑(HOBT), 苯并三氮唑- $N, N, N^{\prime}, N^{\prime}$-四 甲基嫝六氟磷酸盐(HBTU)，4-二甲氨基吡啶(DMAP), 成都诚诺新技术有限公司; 三氟乙酸(TFA), 三异丙基 硅烷(TIS), 甲基三氯硅烷, 阿拉丁公司; 二苯亚砜, 萨 恩化学技术(上海)有限公司; 三羟甲基氨基甲烷(TRIS), SIGMA 公司; 乙腈, Fisher Scientiffic 公司; $N, N$-二异丙 基乙胺(DIEA)，二氯甲烷(DCM), N,N-二甲基甲酰胺 (DMF), 哌啶等其它试剂均为分析纯, 上海国药集团化 学试剂有限公司.

\section{2 实验方法}

\subsection{1 多肽的合成}

第一个氨基酸与树脂的偶联: 称取 Wang 树脂 $(1 \mathrm{~g}$, $0.72 \mathrm{mmol} / \mathrm{g}$ ) 于 $25 \mathrm{~mL}$ 固相反应器中, 用 $15 \mathrm{~mL} \mathrm{DCM}$ 溶 胀 2 h. 称取 Fmoc-Tyr $(t \mathrm{Bu})(0.99 \mathrm{~g}, 2.16 \mathrm{mmol}) 、 \mathrm{HBTU}$ $(0.82 \mathrm{~g}, 2.16 \mathrm{mmol}) 、$ HOBT $(0.29 \mathrm{~g}, 2.16 \mathrm{mmol}) 、$ 催化量 的 DMAP(仅在树脂与第一个氨基酸偶联时添加)溶于 15 $\mathrm{mL}$ DMF 中, 加入 DIEA (1.82 mL, $11.04 \mathrm{mmol})$ 活化后加 入固相反应器中, 在室温下摇床震荡反应 $3 \mathrm{~h}$. 加入 $V$ (乙酸酎) $: V$ (吡啶) $: V(\mathrm{DMF})=2: 1: 3(1 \times 30 \mathrm{~min})$ 进 行封闭, 依次用 DMF, DCM, DMF 洗涤树脂 4 次, 每次 1 min.

剩余氨基酸的偶联: 按照利那洛肽的氨基酸序列, 重复上述步骤进行偶联和脱 Fmoc, 每接完一个氨基酸 以后, 用 Kasier 试剂进行检测, 脯氨酸(Pro)用澳酚兰法 检测, 显色反应为阴性则进入下一个偶联循环, 否则重 复偶联步骤。

多肽的裂解: 完成偶联后, 称取多肽树脂放入反应 瓶中, 按照 $1 \mathrm{~g}$ 树脂 $5 \mathrm{~mL}$ 裂解剂的比例配置裂解液, 在 氮气保护下加入 TFA-TIS- $\mathrm{H}_{2} \mathrm{O}(V: V: V=95: 2.5$ : $2.5)$, 裂解 $2 \mathrm{~h}$ 后抽滤, 加少量 TFA 和 DCM 洗涤 3 次, 合 并滤液, 将得到的滤液用氮气吹去 $3 / 4$ 体积后, 滴入大 量的冰乙醚中析出白色絮状沉淀, 离心得到相应的粗
肽.

\section{2 .2 多肽的纯化及分析}

将得到的粗肽溶于少量水，用 Waters 600 半制备液 相, XB-C18 制备柱进行纯化. 高效液相条件，流动相 A: 水(含体积分数为 $0.1 \%$ 的 TFA); 流动相 B: 乙腈(含体积 分数为 $0.1 \%$ 的 TFA); 流动相梯度：流动相 B $15 \%$ $50 \%$ (体积分数), $20 \mathrm{~min}$; 流速为 $3 \mathrm{~mL} / \mathrm{min}$; 检测波长 $220 \mathrm{~nm}$. 收集主峰, 旋转蒸发除去乙腈, 冻干得到多肽 纯品.

用 Waters 600 半制备液相, C18 分析柱进行分析.高 效液相条件, 流动相 $\mathrm{A}$ : 水 (含体积分数为 $0.1 \%$ 的 TFA); 流动相 B: 乙腈 (含体积分数为 $0.1 \%$ 的 TFA); 流动相梯 度：流动相 B 15\% 50\%(体积分数), $30 \mathrm{~min}$; 流速为 $1 \mathrm{~mL} / \mathrm{min}$; 柱温: $40{ }^{\circ} \mathrm{C}$; 检测波长 $220 \mathrm{~nm}$. 多肽转化率 的计算: 按照产物的 HPLC 在 $220 \mathrm{~nm}$ 的色谱峰面积进 行计算.

\section{2 .3 二硫键的形成}

氯化血红素催化氧化形成二硫键: 称取脱除 Trt 保 护基的利那洛肽线性前体肽 $(3 \mathrm{mg}, 2 \mu \mathrm{mol})$, 溶于 $\mathrm{pH}=$ 8.0 的 Tris- $\mathrm{HCl}$ 的缓冲溶液 $(5 \mathrm{~mL}$ )中, 加入 $20 \%$ (物质的 量比)的氯化血红素(含少量 DIEA)，室温敞口搅拌 $2 \mathrm{~h}$, 反应结束用 HPLC 分析.

$\mathrm{PhS}(\mathrm{O}) \mathrm{Ph} / \mathrm{CH}_{3} \mathrm{SiCl}_{3}$ 脱 $\mathrm{Acm}$ 保护基并形成二硫键: 称取多肽 $(0.6 \mathrm{mg}, 0.4 \mu \mathrm{mol})$, 溶于 TFA $(0.4 \mathrm{~mL})$ 中, 加入 $\mathrm{CH}_{3} \mathrm{SiCl}_{3}(12 \mu \mathrm{L}, 250$ equiv.), $\mathrm{PhS}(\mathrm{O}) \mathrm{Ph}(0.8 \mathrm{mg}, 10$ equiv.), 苯甲醚( $4.3 \mu \mathrm{L}, 100$ equiv.), 室温敞口搅拌 20 $\min$ 加入干燥乙醚 $(10 \mathrm{~mL}), 4 \mathrm{~mol} / \mathrm{L}$ 醋酸 $(5 \mathrm{~mL}$ )震荡, 取 水相进行 HPLC 分析并分离.

辅助材料 (Supporting Information) 合成过程中的 HPLC 分析色谱图及利那洛肽前体肽的质谱. 这些材料 可以免费从本刊网站(http://sioc-journal.cn/)上下载.

\section{References}

[1] Cambridge, M. A. US 7304036, 2007.

[2] Harris, L. A.; Crowell, D. Curr. Opin. Mol. Ther. 2007, 9, 403.

[3] Andresen, V.; Camilleri, M. Drugs Future 2008, 33, 570.

[4] Wu Q. L.; Liu Z. G.; Fu C.; Lin Y. B.; Dai Q. Y. Chin. J. Org. Chem. 2010, 30, 1517 (in Chinese) (吴巧玲，刘珠果，付超，林原斌，戴秋云，有机化学， 2010，30, 1517.)

[5] Eliasen, R.; Andresen, T. L.; Conde-Frieboes, K. W. Peptides 2012, 34,144

[6] Dekan, Z.; Mobli, M.; Pennington, M. W.; Fung, E.; Nemeth, E.; Alewood, P. F. Angew. Chem., Int. Ed. 2014, 53, 1.

[7] Veber, D. F.; Milkowski, J. D.; Varga, S. L.; Denkewalter, R. G.; Hirschmann, R. J. Am. Chem. Soc. 1972, 94, 5456.

[8] Munson, M. C.; Barany, G. J. Am. Chem. Soc. 1993, 115, 10203

[9] Kawakami, T.; Aimoto, S. Tetrahedron Lett. 1998, 39, 7901.

[10] Hunter, M. J.; Komives, E. A. Anal. Biochem. 1995, 228, 173.

[11] Miriam, G. B.; Judit, T. P.; Marta, P. B.; Marta, P. B.; Oleg, W.; 
Matthieu, G.; Fernando, A. Biopolymers 2011, 96, 69.

[12] Ge, W. W.; Chen, J.; Zong, L.; Li, J.; Sui, S. H.; Wu, W. H.; Zhang, M.; Dong, J. J. Chem. J. Chin. Univ. 2017, 38, 1052 (in Chinese). (葛巍巍, 陈静, 宗良, 李建, 隋少卉, 吴为辉, 张鸣, 董俊军, 高等学校化学学报, 2017, 38, 1052.)

[13] Akaji, K.; Tatsumi, T.; Yoshida, M.; Kimura, T.; Fujiwara, Y.; Kiso, Y. J. Am. Chem. Soc. 1992, 114, 4137.
[14] Ildikó, S.; Gitta, S.; Ference, H.; Gábor, M. Biopolymers 2006, 88 , 20.

[15] Ge, W. W.; Chen, J.; Zhang, Y.; Zong, L.; Zhang, M.; Dong, J. J. J. Int. Pharm. Res. 2017, 44, 585 (in Chinese).

(葛巍巍, 陈静, 张也, 宗良, 张鸣, 董俊军, 国际药学研究杂志, 2017, 44, 585.)

(Zhao, X.) 